Cite this article as: BMJ, doi:10.1136/bmj.38547.638183.06 (published 15 August 2005)

\title{
Papers
}

\section{Self reported stress and risk of breast cancer: prospective cohort study}

Naja Rod Nielsen, Zuo-Feng Zhang, Tage S Kristensen, Bo Netterstrøm, Peter Schnohr, Morten Grønbæk

\begin{abstract}
Objective To assess the relation between self reported intensity and frequency of stress and first time incidence of primary breast cancer.

Design Prospective cohort study with 18 years of follow-up. Setting Copenhagen City heart study, Denmark.

Participants The 6689 women participating in the

Copenhagen City heart study were asked about their perceived level of stress at baseline in 1981-3. These women were followed until 1999 in the Danish nationwide cancer registry, with $<0.1 \%$ loss to follow-up.
\end{abstract}

Main outcome measure First time incidence of primary breast cancer.

Results During follow-up 251 women were diagnosed with breast cancer. After adjustment for confounders, women with high levels of stress had a hazard ratio of 0.60 (95\% confidence interval 0.37 to 0.97 ) for breast cancer compared with women with low levels of stress. Furthermore, for each increase in stress level on a six point stress scale an $8 \%$ lower risk of primary breast cancer was found (hazard ratio $0.92,0.85$ to 0.99 ). This association seemed to be stable over time and was particularly pronounced in women receiving hormone therapy.

Conclusion High endogenous concentrations of oestrogen are a known risk factor for breast cancer, and impairment of oestrogen synthesis induced by chronic stress may explain a lower incidence of breast cancer in women with high stress. Impairment of normal body function should not, however, be considered a healthy response, and the cumulative health consequences of stress may be disadvantageous.

\section{Introduction}

Breast cancer is the most common cancer in women in terms of both incidence and prevalence. ${ }^{1}$ It is a hormone dependent disease with a clear positive relation to high endogenous concentrations of oestrogen. ${ }^{2}$ The role of stress in the aetiology of breast cancer has been an area of emerging interest, partly because of the ability of stress to affect the hormonal system and especially oestrogen synthesis. ${ }^{3-7}$ A potential relation between stress and risk of breast cancer has been examined in studies with different designs and with conflicting results. ${ }^{8-15}$ Different measures of stress, as applied in these studies, may well have different physiological and psychological impacts. Death of a spouse or near relative is a major acute stressor, whereas stress experienced in daily life is more moderate and chronic in nature.

The risk of breast cancer associated with the acute stress of major life events has been assessed in several studies, ${ }^{9-11}{ }^{13}$ but less attention has been given to the effect of perceived daily stress. ${ }^{12} 1415$ Prolonged low key stress of everyday life results in a persistent activation of stress hormones, which may impair oestrogen synthesis, ${ }^{16}$ and may thereby be related to a lower risk of breast cancer. Everyday stress may also indirectly affect the risk of breast cancer through changes in health related behaviour. This study provides an important opportunity to explore the impact of everyday stress on the long term risk of first time incidence of primary breast cancer among 6689 women prospectively followed up for 18 years.

\section{Methods}

Study population-The Copenhagen City heart study is a longitudinal study initiated in 1976. An age stratified random sample of 19698 Danish men and women were invited to participate. All participants gave written informed consent. In 1981-3 the same population had additional examinations. The second examination of 7018 women included questions on stress and is therefore used as baseline for the study reported here. The response rate was $70 \%$. We excluded women with breast cancer before baseline $(n=120)$ or lacking information on stress or other covariates $(n=209)$, leaving 6689 women. Twenty six $(<0.1 \%)$ women were lost to follow-up. A detailed description of the Copenhagen City heart study has previously been published. ${ }^{17}$

Everyday stress-The study participants were asked about their level of stress in terms of intensity and frequency. In the questionnaire, stress was exemplified as the sensation of tension, nervousness, impatience, anxiety, or sleeplessness. Participants were asked to report their stress intensity as none (0), light (1), moderate (2), or high (3). Frequency of stress was reported as never/hardly ever (0), monthly (1), weekly (2), or daily (3). ${ }^{18}$ We added the scores of the two questions and combined them into a continuous stress score from 0 to 6 . We categorised the stress score into low (0-1 points), medium (2-4 points), and high (5-6 points) stress in order to examine differences in incidence of breast cancer for low and high levels of stress.

Covariates-We considered the following variables as potential confounders for the analyses: current oral contraceptive use (yes/no), other hormone therapy (yes/no), menopause at baseline (yes/no), body mass index (continuous), number of children $(0,1-2, \geq 3)$, physical activity in leisure time (low, medium, high), alcohol consumption (0 drinks/week, 1-14 drinks/week, $>14$ drinks/week), and education ( $<8$ years, $8-11$ years, $\geq 12$ years).

Follow-up-We followed participants from the date of the second examination until the date of first diagnosis of primary breast cancer $(n=251)$, death $(n=2224)$, emigration $(n=26)$, or the end of follow-up on 31 December $1999(n=4188)$. We used 
Table 1 Baseline characteristics of women participating in the second examination of the Copenhagen City heart study in 1981-3. Values are numbers (percentages) unless stated otherwise

\begin{tabular}{|c|c|c|c|c|}
\hline \multirow{3}{*}{ Participants } & \multirow{3}{*}{$\begin{array}{c}\text { Study population } \\
6689\end{array}$} & \multicolumn{3}{|c|}{ Stress* } \\
\hline & & Low & Medium & High \\
\hline & & $2823(42)$ & 3201 (48) & $665(10)$ \\
\hline Mean (SD) age (years) & $57(12)$ & $58(12)$ & $55(12)$ & $58(12)$ \\
\hline Premenopausal & $1766(26)$ & 672 (24) & 978 (31) & $116(17)$ \\
\hline $\begin{array}{l}\text { Current oral } \\
\text { contraceptive users }\end{array}$ & $250(4)$ & $99(4)$ & $143(4)$ & $8(1)$ \\
\hline Other hormone therapy & $1045(16)$ & $328(12)$ & $568(18)$ & $149(22)$ \\
\hline Mean (SD) No of children & $1.6(1.3)$ & $1.6(1.4)$ & $1.6(1.3)$ & $1.7(1.4)$ \\
\hline $\begin{array}{l}\text { Mean (SD) body mass } \\
\text { index }\left(\mathrm{kg} / \mathrm{m}^{2}\right)\end{array}$ & $25(5)$ & $25(5)$ & $25(4)$ & $25(5)$ \\
\hline $\begin{array}{l}\text { High alcohol } \\
\text { consumption }\end{array}$ & $449(7)$ & $152(5)$ & $230(7)$ & $67(10)$ \\
\hline Low education & 3096 (46) & 1340 (47) & 1388 (43) & 368 (55) \\
\hline Physically inactive & $1179(18)$ & $442(16)$ & $520(16)$ & $217(33)$ \\
\hline
\end{tabular}

* Participants reported stress intensity and frequency on a standard questionnaire with four multiple choice categories (0-3 points) for each stress measure. The scores of the two questions were added and combined into a continuous stress score from 0 to 6 . This stress score was categorised into low (0-1 points), medium (2-4 points), and high (5-6 points).

the civil registry number, which is unique to every Danish citizen, to identify primary breast cancer events through linkage to the Danish national cancer registry, which contains data on all cancer diagnoses in Denmark. We used ICD-7 codes170.0-170.5, $470.0-470.5$, and $870.0-870.2$ to identify cases of primary invasive breast cancer. We followed the vital status of the study population in the central death registry. Information on diagnosis of breast cancer was updated until 1999, making it possible to follow the participants from the second examination for 16-18 years for a primary diagnosis of breast cancer.

Statistical methods-We used Cox regression models (SAS/ STAT software version 8.2) to analyse data with age as the time scale. All included variables met the assumption of proportional hazards. Initially, we estimated the age adjusted hazard ratio of primary breast cancer associated with stress intensity, stress frequency, and stress score (continuous and in categories of low, medium, and high stress). Subsequently, we fitted a multivariate Cox regression model according to the "change in estimate" method, ${ }^{19}$ with a cut-off point of $5 \%$ change, to adjust for potential confounding from baseline covariates. We used trend analyses to assess dose-response relations between stress and breast cancer. We did a $\chi^{2}$ test for goodness of fit before including any variables as continuous and also used it to test for linear trends. To estimate the effect of prolonged follow-up, we assessed the association in the first and last nine years of follow-up. Finally, we did subgroup analyses to assess potential effect modification.

\section{Results}

Baseline characteristics-Mean age at baseline was 57 (range 21-91) years. Ten per cent of the women reported high levels of stress (table 1). Mean age, number of children, and body mass index were similar at the different stress levels. A lower proportion of highly stressed women than less stressed women were premenopausal and used oral contraceptives. A higher proportion of the women in the high stress group received hormone therapy, had low education and high alcohol intake, and were physically inactive in their leisure time compared with women with lower levels of stress. During follow-up, 251 cases of primary breast cancer occurred. A higher percentage of women in the high stress $(n=261,39.3 \%)$ than in the medium stress $(\mathrm{n}=972,30.4 \%)$ or low stress group $(\mathrm{n}=991,35.1 \%)$ died during follow-up.
Table 2 Incidence and hazard ratio of primary breast cancer associated with intensity and frequency of stress among 6689 Danish women participating in the second examination of the Copenhagen City heart study in 1981-3

\begin{tabular}{|c|c|c|c|c|}
\hline & $\begin{array}{l}\text { No of } \\
\text { breast } \\
\text { cancers }\end{array}$ & $\begin{array}{l}\text { Incidence per } \\
100000 \\
\text { person years }\end{array}$ & $\begin{array}{c}\text { Age adjusted } \\
\text { hazard ratio (95\% } \\
\text { CI) }\end{array}$ & $\begin{array}{l}\text { Multi-adjusted }^{*} \\
\text { hazard ratio (95\% } \\
\text { CI) }\end{array}$ \\
\hline \multicolumn{5}{|l|}{ Stress intensity } \\
\hline None $(n=2214)$ & 96 & 292 & 1 (reference) & 1 (reference) \\
\hline Light $(\mathrm{n}=2608)$ & 97 & 243 & 0.89 (0.67 to 1.18$)$ & 0.85 (0.64 to 1.13$)$ \\
\hline Moderate $(n=1384)$ & 44 & 210 & 0.74 (0.52 to 1.05$)$ & 0.68 (0.47 to 0.98$)$ \\
\hline High $(n=483)$ & 14 & 203 & 0.65 (0.37 to 1.13$)$ & $0.61(0.35$ to 1.07$)$ \\
\hline$P$ value for trend & & & 0.04 & 0.02 \\
\hline \multicolumn{5}{|l|}{ Stress frequency } \\
\hline Never $(n=2854)$ & 119 & 282 & 1 (reference) & 1 (reference) \\
\hline Monthly ( $n=1994)$ & 71 & 228 & 0.90 (0.67 to 1.20$)$ & 0.85 (0.64 to 1.15$)$ \\
\hline Weekly $(\mathrm{n}=1168)$ & 40 & 227 & 0.83 (0.58 to 1.19$)$ & 0.78 (0.55 to 1.13$)$ \\
\hline Daily $(\mathrm{n}=673)$ & 21 & 213 & 0.70 (0.44 to 1.11$)$ & 0.67 (0.42 to 1.07$)$ \\
\hline $\mathrm{P}$ value for trend & & & 0.09 & 0.06 \\
\hline
\end{tabular}

*Adjusted for age, current oral contraceptive use, other hormone therapy, menopausal status, number of children, body mass index, alcohol consumption, physical activity in leisure time, and education.

Stress intensity, stress frequency, and risk of breast cancer-Seven per cent of the women reported high stress intensity, and 10\% reported high frequency of stress. The adjusted hazard ratio of primary breast cancer seemed to be inversely associated with both stress intensity (test for trend, $\mathrm{P}=0.02$ ) and stress frequency (test for trend, $\mathrm{P}=0.06$ ) (table 2).

Stress score and risk of breast cancer-After adjustment for potential confounders, an $8 \%$ reduction (hazard ratio $0.92,95 \%$ confidence interval 0.85 to 0.99 ) in risk of primary breast cancer occurred for each increase in stress level on the six point stress scale (table 3). Higher stress was inversely associated with incidence of primary breast cancer (test for trend, $\mathrm{P}=0.02$ ), and high stress was associated with a hazard ratio of $0.60(0.37$ to 0.97 ) for breast cancer compared with low stress.

Subgroup analyses-One hundred and fourteen first time primary breast cancers occurred in the first nine years of followup, and 137 cases occurred in the last nine years. The relative effect of stress on incidence of breast cancer seemed to be similar in the two periods of follow-up (table 4). Sixteen per cent $(\mathrm{n}=1045)$ of the women were receiving hormone therapy at baseline, and the effect of stress on risk of breast cancer seemed to be mainly confined to these women (table 5). The $\mathrm{P}$ value for effect modification was 0.09 . Among women receiving hormone therapy, the hazard ratio for primary breast cancer was $0.83(0.72$ to 0.97 ) for each increase in stress level on the six point stress

Table 3 Incidence and hazard ratio of primary breast cancer associated with stress score among 6689 Danish women participating in the Copenhagen City heart study in 1981-3

\begin{tabular}{|c|c|c|c|c|}
\hline Stress score & $\begin{array}{l}\text { No of } \\
\text { breast } \\
\text { cancers }\end{array}$ & $\begin{array}{c}\text { Incidence per } \\
100000 \text { person } \\
\text { years }\end{array}$ & $\begin{array}{c}\text { Age adjusted } \\
\text { hazard ratio (95\% } \\
\text { CI) }\end{array}$ & $\begin{array}{l}\text { Multi-adjusted }^{\star} \\
\text { hazard ratio (95\% } \\
\text { CI) }\end{array}$ \\
\hline Continuous & & & 0.93 (0.87 to 1.00$)$ & $0.92(0.85$ to 0.99$)$ \\
\hline \multicolumn{5}{|l|}{ Categorised: } \\
\hline $\begin{array}{l}\text { Low stress } \\
(\mathrm{n}=2823)\end{array}$ & 120 & 285 & 1 (reference) & 1 (reference) \\
\hline $\begin{array}{l}\text { Medium stress } \\
(\mathrm{n}=3201)\end{array}$ & 112 & 229 & 0.84 (0.65 to 1.09$)$ & 0.80 (0.62 to 1.04$)$ \\
\hline $\begin{array}{l}\text { High stress } \\
(n=665)\end{array}$ & 19 & 194 & 0.63 (0.39 to 1.02) & $0.60(0.37$ to 0.97$)$ \\
\hline $\mathrm{P}$ value for trend & & & 0.04 & 0.02 \\
\hline
\end{tabular}

${ }^{*}$ Adjusted for age, current oral contraceptive use, other hormone therapy, menopausal status, number of children, body mass index, alcohol consumption, physical activity in leisure time, and education. 
Table 4 Incidence and hazard ratio of primary breast cancer associated with categorised stress score among 6689 Danish women participating in the Copenhagen City heart study in 1981-3, according to time period of follow-up

\begin{tabular}{|c|c|c|c|c|}
\hline & \multicolumn{2}{|c|}{ First nine years of follow-up } & \multicolumn{2}{|c|}{ Last nine years of follow-up } \\
\hline & $\begin{array}{c}\text { Incidence per } 100000 \text { person } \\
\text { years }\end{array}$ & Hazard ratio* $(95 \% \mathrm{Cl})$ & $\begin{array}{c}\text { Incidence per } 100000 \text { person } \\
\text { years }\end{array}$ & Hazard ratio* $(95 \% \mathrm{Cl})$ \\
\hline Low stress & 239 & 1 (reference) & 339 & 1 (reference) \\
\hline Medium stress & 190 & 0.82 (0.55 to 1.21$)$ & 272 & $0.78(0.55$ to 1.11$)$ \\
\hline High stress & 167 & 0.63 (0.31 to 1.28$)$ & 227 & $0.60(0.30$ to 1.16$)$ \\
\hline$P$ value for trend & & 0.14 & & 0.07 \\
\hline
\end{tabular}

*Adjusted for age, current oral contraceptive use, other hormone therapy, menopausal status, number of children, body mass index, alcohol consumption, physical activity in leisure time, and education.

scale. No notable effect modification occurred in subgroups of menopausal status, physical activity, alcohol consumption, oral contraceptive use, education, and number of children (data not shown).

\section{Discussion}

Among 6689 women followed up for an average of 18 years, higher self reported everyday stress was associated with lower risk of breast cancer. Our results are similar to those from the nurses' health study, in which Kroenke et al found self reported stress from adult care giving to be associated with lower incidence of breast cancer. ${ }^{14}$ Our results are, however, in contrast with the results of a Finnish cohort study, which found no association between stress of daily activities and breast cancer, and a Swedish study in which severe mental stress was associated with higher incidence of breast cancer. ${ }^{12}{ }^{15}$ Some of the discrepancy may be explained by the fact that different measures of stress were applied and that the Finnish and Swedish studies included all incident cases of breast cancer, whereas we confined our analyses to first time incidence of primary breast cancer. Apart from these studies, the main focus has been on stressful life events. However, the nature of sustained everyday stress is different from stressful life events, and a greater risk of breast cancer associated with stressful life events is not necessarily in contrast with a lower risk of breast cancer associated with daily stress.

\section{Strengths and weaknesses}

The prospective design of the Copenhagen City heart study ensured temporality between stress and incidence of breast cancer. The cohort is a large random sample of the general population of Copenhagen. Furthermore, linkage of civil registry numbers to nationwide population based registers enabled identification of virtually all cases of breast cancer and allowed for nearly complete long term follow-up.

Information on several important risk factors for breast cancer, such as family history of breast cancer, age at menarche, and age at first full time pregnancy, was not obtained. However, to confound the results these factors would also have to be related to stress. We cannot exclude that having experienced breast cancer in a near family member may act as a stressor and thereby

\section{Table 5 Hazard ratio of primary breast cancer associated with stress score among 6689 Danish women participating in the Copenhagen City heart study in 1981-3, in subgroups of hormone therapy}

\begin{tabular}{lccc} 
Stress score & $\begin{array}{c}\text { No of breast } \\
\text { cancers }\end{array}$ & $\begin{array}{c}\text { Age adjusted hazard } \\
\text { ratio }(95 \% \text { Cl) }\end{array}$ & $\begin{array}{c}\text { Multi-adjusted* hazard } \\
\text { ratio }(\mathbf{9 5 \%} \mathbf{~ C l})\end{array}$ \\
\hline $\begin{array}{l}\text { No hormone therapy } \\
(\mathrm{n}=5644)\end{array}$ & 184 & $0.95(0.87$ to 1.04$)$ & $0.96(0.88$ to 1.05$)$ \\
$\begin{array}{l}\text { Hormone therapy } \\
(\mathrm{n}=1045)\end{array}$ & 67 & $0.82(0.71$ to 0.95$)$ & $0.83(0.72$ to 0.97$)$ \\
\hline
\end{tabular}

*Adjusted for age, current oral contraceptive use, menopausal status, number of children, body mass index, alcohol consumption, physical activity in leisure time, and education. lead to higher levels of stress, which would result in a spurious positive association between stress and breast cancer. This is opposite to the inverse association we observe in our study and therefore cannot explain it. Late age of menarche would have to be relatively strongly related to high stress in order to explain our results, but we know of no empirical evidence or biological explanation that would support such an expectation. Late age at first full term pregnancy is a well established risk factor for breast cancer. More women with high stress had low education (55\% versus $47 \%$ in the low stress group), and women with low education tend to have their first pregnancy earlier than other women. Thus, we cannot exclude that some of the observed inverse association is due to uncontrolled confounding by age at first pregnancy. However, adjustment for number of children, which also tends to be correlated with age at first pregnancy, as well as adjustment for socioeconomic status only slightly changed the estimates.

Mammographic screening for women aged 50-69 years was introduced in Copenhagen in 1991. ${ }^{20}{ }^{21}$ Stressed women may have been less likely to participate in the screening programme and may therefore have had invasive breast cancer diagnosed at a later stage. According to Olsen et al, an expected increase in incidence of invasive breast cancer occurred after the first screening round, but the incidence dropped to the pre-screening level in the following screening rounds, indicating that over-diagnosis of breast cancer was not a major problem. ${ }^{21}$ Furthermore, screening took place only in women aged 50-69 years and in the last nine years of our study. The effect estimates were similar in the first and last nine years of follow-up (table 4), so bias by screening is unlikely to explain our results.

How stress is to be defined and measured remains a point of debate. So far the literature has focused on external stressors with less emphasis given to how they are perceived by the individual. Each person, however, has different capacity and ways of coping with stressful situations, and the same external stressor may result in different levels of perceived stress. A measure of perceived stress will therefore provide a better measure of the actual level of stress experienced by the individual as opposed to a count of potential stressful situations defined by the researcher.

More women in the high stress group (39.3\%) than in the medium stress $(30.4 \%)$ and low stress $(35.1 \%)$ groups died during follow-up. Although this indicates no systematic difference, it may raise concern about how censoring has influenced the results. We assumed, in the statistical model, that censoring was independent of breast cancer risk within each stratum of stress. Competing causes, such as death from cardiovascular disease, could, however, be associated with risk of breast cancer within strata of stress owing to other common risk factors. Some of the common risk factors, such as socioeconomic status, have opposite effects on the two diseases, whereas other risk factors, such as low physical activity, increase the risk of both diseases. On average, we would expect the bias to level out and 
the average risk of breast cancer among women not censored to be similar to what it would have been if no such censoring had occurred. Thus, although our results may have been influenced by bias from non-independent censoring, we find it unlikely that this could fully explain them.

\section{Possible causal pathways between perceived stress and breast cancer}

In most cases, the physiological effects of acute stressors are reversible owing to the amazing ability of the human organism to re-establish allostasis. The problems arise when the stress response becomes chronic and results in permanent disturbances. In a normally functioning female reproductive system, the hypothalamus releases luteinising hormone releasing hormone, which stimulates the pituitary gland to release luteinising hormone and follicle stimulation hormone. Luteinising hormone stimulates the ovaries to synthesise oestrogens, whereas follicle stimulation hormone stimulates the ovaries to release eggs. This is called the hypothalamic-pituitary-gonadal axis. Stress can affect the signals of this axis by activating the hypothalamic-pituitary-adrenal axis and the sympathetic nervous system. Several studies in mammals have found that activation of the hypothalamic-pituitary-adrenal axis inhibits the function of the hypothalamic-pituitary-gonadal axis and thereby decreases oestrogen synthesis, but data in humans are sparse. ${ }^{3-6}{ }^{16}$ However, in a recent study in which care giving was used as an indicator of chronic stress, significantly lower concentrations of bioavailable oestradiol were found among female care givers than among non-care givers. ${ }^{14}$ In essence, stress induced suppression of oestrogen secretion could explain a reduced risk of breast cancer.

An imbalance in the allostatic concentration of reproductive hormones can also result in other reproductive disturbances and mood swings and initiate depression in susceptible women. ${ }^{22}{ }^{23}$ Some women may be more sensitive to hormonal disturbances and therefore also more likely to receive hormone therapy to lessen their symptoms. Hormone sensitive women are more likely to be susceptible to stress induced changes in oestrogen synthesis, which could explain why stress mainly seems to be associated with lower risk of breast cancer among women receiving hormone therapy.

\section{Conclusions}

It is biologically plausible that the lower risk of breast cancer associated with stress observed in this long term prospective cohort study could be due to stress induced imbalances in normal concentrations of oestrogens. However, stress induced disturbances in allostasis cannot be considered a healthy response, and prolonged stress may have harmful effects on a range of other diseases, especially cardiovascular diseases.

We thank the staff and the participants of the Copenhagen City heart study. Contributors: NRN contributed to the conception and design of the study, the analysis and interpretation of data, and the drafting the paper. Z-FZ, TSK, BN, and MG contributed to the conception and design of the study and to critically revising the paper. PS contributed to the design of the study, the acquisition of data, and critically revising the paper. TSK designed the stress questions used in the study. All authors approved the final version of the article. MG is the guarantor.

Competing interests: None declared.

Funding: This study was supported by funds from the Health Insurance Foundation.

Ethical approval: The Danish ethics committee for the City of Copenhagen and Frederiksberg approved the study (\#100.2039/91).

1 Parkin DM, Bray FI, Devesa SS. Cancer burden in the year 2000: the global picture. Eur J Cancer 2001;37:S4-66.

\section{What is already known on this topic}

A potential relation between stress and risk of breast cancer has been examined in studies with different designs and conflicting results

The risk of breast cancer associated with the acute stress of major life events has been assessed in several studies, but less attention has been given to the effect of perceived daily stress

\section{What this study adds}

Higher levels of everyday stress are associated with lower incidence of primary breast cancer in a dose-response manner among middle aged women

Women who receive hormone replacement therapy seem to be most susceptible to this effect

Stress induced disturbances cannot be considered a healthy response, and the cumulative health consequences of stress may be disadvantageous

2 Hankinson S, Hunter D. Breast cancer. In: Adami H, Hunder D, Trichopoulos D, eds. Textbook of cancer epidemiology. New York: Oxford University Press, 2002:301-39. Rivier C. Luteinizing-hormone-releasing hormone, gonadotropins, and gonadal steroids in stress. Ann N Y Acad Sci 1995;771:187-91.

Sapolsky R. Sex and reproduction. In: Sapolsky R, ed. Why zebras don't get ulcers. New York: W H Freeman and Company, 1998:101-25.

5 Negro-Vilar A. Stress and other environmental factors affecting fertility in men and Negro-Vilar A. Stress and other environmental factors
women: overview. Environ Health Perspect 1993;101:59-64

6 Breen KM, Karsch FJ. Does cortisol inhibit pulsatile luteinizing hormone secretion at the hypothalamic or pituitary level? Endocrinology 2004;145:692-8.

7 McEwen BS. Protective and damaging effects of stress mediators. N Engl J Med 1998;338:171-9.

Achat H, Kawachi I, Byrne C, Hankinson S, Colditz G. A prospective study of job strain and risk of breast cancer. Int J Epidemiol 2000;29:622-8.

9 Duijts SF, Zeegers MP, Borne BV. The association between stressful life events and breast cancer risk: a meta-analysis. Int J Cancer 2003;107:1023-9.

10 Lillberg K, Verkasalo PK, Kaprio J, Teppo L, Helenius H, Koskenvuo M. Stressful life events and risk of breast cancer in 10,808 women: a cohort study. Am J Epidemiol 2003;157:415-23.

11 Protheroe D, Turvey K, Horgan K, Benson E, Bowers D, House A. Stressful life events and difficulties and onset of breast cancer: case-control study. BMJ 1999:319:1027-30.

12 Lillberg K, Verkasalo PK, Kaprio J, Teppo L, Helenius H, Koskenvuo M. Stress of daily activities and risk of breast cancer: a prospective cohort study in Finland. Int J Cancer 2001;91:888-93

13 Johansen C, Olsen JH. Psychological stress, cancer incidence and mortality from nonmalignant diseases. Br J Cancer 1997;75:144-8.

14 Kroenke CH, Hankinson SE, Schernhammer ES, Colditz GA, Kawachi I, Holmes MD. Caregiving stress, endogenous sex steroid hormone levels, and breast cancer incidence. Am J Epidemiol 2004;159:1019-27.

15 Helgesson O, Cabrera C, Lapidus L, Bengtsson C, Lissner L. Self-reported stress levels predict subsequent breast cancer in a cohort of Swedish women. Eur J Cancer Prev 2003;12:377-81.

16 Ferin M. Clinical review 105: stress and the reproductive cycle. J Clin Endocrinol Metab 1999;84:1768-74.

17 Appleyard M, Hansen AT, Schnohr P, Jensen G, Nyboe J. The Copenhagen City heart study: a book of tables with data from the first examination (1976-78) and a five year follow-up (1981-83). Scand J Soc Med 1989;170:1-160.

18 Truelsen T, Nielsen N, Boysen G, Gronbaek M. Self-reported stress and risk of stroke: the Copenhagen City heart study. Stroke 2003;34:856-62.

19 Greenland S, Rothman K. Introduction to stratified analysis. In: Rothman K, Greenland S, eds. Modern epidemiology. Philadelphia: Lippincott Williams \& Wilkins, 1998:253-300.

20 Olsen AH, Jensen A, Njor SH, Villadsen E, Schwartz W, Vejborg I, et al. Breast cancer incidence after the start of mammography screening in Denmark. $\mathrm{Br} J$ Cancer 2003;88:362-5.

21 Olsen AH, Njor SH, Vejborg I, Schwartz W, Dalgaard P, Jensen MB, et al. Breast cancer mortality in Copenhagen after introduction of mammography screening: cohort study. BMI 2005;330:220.

22 Halbreich U. Role of estrogen in postmenopausal depression. Neurology 1997;48:S16-9.

23 Birkhauser M. Depression, menopause and estrogens: is there a correlation? Maturitas 2002;41:S3-8.

(Accepted 27 June 2005)

doi $10.1136 /$ bmj.38547.638183.06

National Institute of Public Health, Øster Farimagsgade 5A, DK-1399 Copenhagen K, Denmark

Naja Rod Nielsen student and research assistant 
Morten Grønbæk professor

Department of Epidemiology, UCLA School of Public Health, Los Angeles, CA,

USA

Zuo-Feng Zhang professor

National Institute of Occupational Health, Copenhagen

Tage S Kristensen professor
Clinic of Occupational Medicine, Hillerød Hospital, Hillerød, Denmark Bo Netterstrøm research director

Copenhagen City Heart Study, Epidemiological Research Unit, Bispebjerg University Hospital, Copenhagen

Peter Schnohr consultant

Correspondence to: N R Nielsen nrn@niph.dk

س 